\title{
Automatic Material Classification via Proprioceptive Sensing and Wavelet Analysis During Excavation
}

\author{
Unal Artan \\ Ingenuity Labs Research Institute \\ Queen's University \\ Kingston, Canada \\ u.artan@queensu.ca
}

\author{
Heshan Fernando \\ Ingenuity Labs Research Institute \\ Queen's University \\ Kingston, Canada \\ ORCID 0000-0003-2930-8925
}

\author{
Joshua A. Marshall \\ Ingenuity Labs Research Institute \\ Queen's University \\ Kingston, Canada \\ ORCID 0000-0002-7736-7981
}

\begin{abstract}
This paper presents an excavation material classification methodology that uses wavelet analysis and unsupervised learning on acceleration measurements. The technique was validated by using acceleration data that were acquired from three inertial measurement units (IMUs) on an instrumented 1-tonne capacity wheel loader. One IMU was installed on the loader's boom and two on the bucket. The acceleration signals were logged for 32 manual excavation trials in three excavation materials with different rock size distributions, and the data were processed offline. The continuous wavelet transform was applied to the acceleration signals to extract features from the acceleration signals. The results show that classifying the wavelet feature set using an unsupervised $k$-means algorithm provides an average material classification accuracy of $81 \%$, when attempting to simultaneously classify all three materials.
\end{abstract}

Index Terms-mining automation, machine learning, proprioceptive sensing

\section{INTRODUCTION}

Knowledge about excavation material characteristics, such as rock size distributions (i.e., fragmentation), in the mining and aggregates industries can optimize downstream processes, such as crushing and grinding [1], and upstream processes, such as blast design refinement [2]. Furthermore, material identification could also be used to adapt control systems for autonomous excavation [3], [4]. State-of-the-art technologies for fragmentation analysis utilize cameras and image processing methods [5], [6]; however, these technologies are limited by their ability to only see the surface of a rock pile [7]. As well, lighting and calibration requirements make exteroceptive sensing impractical for use in underground operations. Safely capturing the images for analysis requires an operator to pause the excavation process, so image data-and in turn, the fragmentation analysis-is only acquired occasionally. In contrast, utilizing proprioceptive sensors onboard mining equipment that interacts directly with the excavation material (e.g., loaders such as the wheel loader shown in Fig. 1 and haul trucks) could overcome these limitations and also provide fragmentation estimates on a continuous basis, throughout the production process.

This paper presents an excavation material classification methodology that uses only acceleration measurements from

This project was funded in part by the NSERC Canadian Robotics Network (NCRN) under grant number NETGP 508451-17.

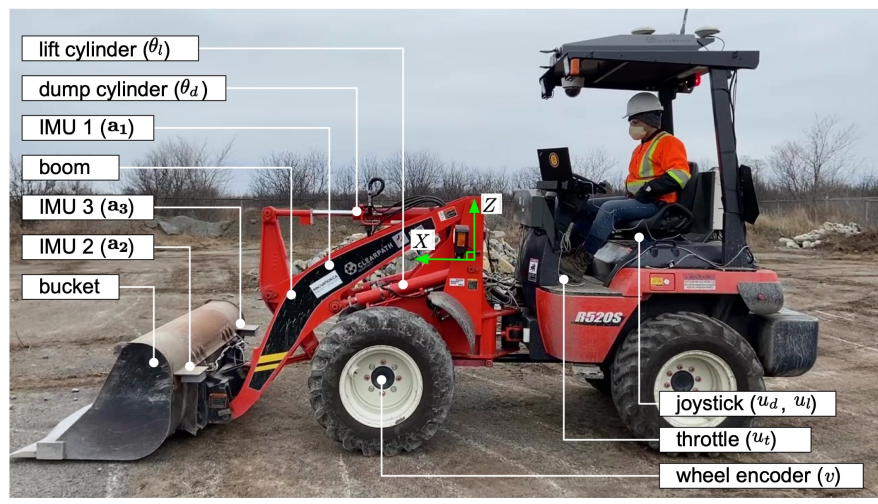

Fig. 1: The Kubota R520s robotic 1-tonne-capacity wheel loader that was used for field experiments. The available sensor signals are described in Table I.

inertial measurement units (IMUs) installed on the boom and bucket of a wheel loader. The methodology involves acquiring acceleration data during the loading operation, extracting features from the acceleration signals using wavelet analysis, and classifying the features using an unsupervised learning algorithm. The methodology was validated by using acceleration data acquired from 32 manual loading operations with an instrumented, 1-tonne capacity, wheel loader (shown in Fig. 1) and three materials with different rock size distributions.

\section{Motivation AND BACKGROUND}

Our previous research in excavation material classification demonstrated the sufficiency of using force data, obtained from autonomous loading experiments with a 14-tonne capacity load-haul-dump machine, for the binary classification of rock and gravel materials [4]. In this paper, the research is extended towards the classification of materials with different rock size distributions, which is useful information for mining and aggregate handling operations, using acceleration data obtained from manual loading with an instrumented wheel loader. Manual loading operations are not repeatable, so they introduce a lot of variability to the data. However, manual loading is more common today as autonomous loading is still an area of research and development. Furthermore, most wheel loaders in operation today are not equipped with the hydraulic 
pressure sensors that were used to acquire force data in [4]. Thus, material classification using acceleration measurements obtained from manual loading operations provide a broader range of applications. The IMUs for acceleration measurements can be installed on any loader or machine that interacts with the material.

Aggregate material classification using acceleration data and wavelet analysis was first investigated in [8], where two IMUs were mounted on a small laboratory-scale haul truck, and acceleration data were acquired as material was dumped on to the bed of the haul truck. Wavelet analysis was used to find distinguishable features from the acceleration signals to identify four different aggregate materials. A similar methodology is utilized in this work for feature extraction from the acceleration signals. The features are classified using $k$ means clustering. The following subsections provide a brief overview of wavelet analysis and $k$-means clustering.

\section{A. Wavelet Analysis}

Wavelet analysis begins with the mother wavelet $\Psi$, which has a zero mean and $L^{2}$ square norm one [9]. The mother wavelet can be scaled by $s$ and translated in time by $\tau$ using

$$
\Psi_{s, \tau}(t)=\frac{1}{\sqrt{s}} \Psi\left(\frac{t-\tau}{s}\right) .
$$

The continuous wavelet transform (CWT) can be expressed as

$$
C W T(s, \tau)=\int_{-\infty}^{\infty} a(t) \Psi_{s, \tau}^{*}(t) d t
$$

were $\Psi^{*}$ denotes the complex conjugate of $\Psi$. The CWT result can be integrated over time to obtain

$$
\beta(s)=\int_{-\infty}^{\infty} C W T(s, \tau) d \tau
$$

In this paper, we use the continuous wavelet transform function in MATLAB ${ }^{\circ}$, which provides an approximate frequency (pseudo-frequency). The result $C W T(s, \tau)$ is mapped to $C W T(f, \tau)$, which means $\beta(s)$ is mapped to $\beta(f)$.

\section{B. k-Means Classifier}

We employ the $k$-means unsupervised learning algorithm, which partitions $n$ observations (i.e., features) $\boldsymbol{o}_{1}, \boldsymbol{o}_{2}, \ldots, \boldsymbol{o}_{n}$ into $k$ clusters [10]. To begin, $k$ centroids (or means) $\boldsymbol{\mu}_{1}, \boldsymbol{\mu}_{2}, \ldots, \boldsymbol{\mu}_{k}$ are initialized in the input space. We do this by randomly choosing $k$ observations from the data set. The value of $k$ may be assigned based on the final application (i.e., the number of desired classes). In this work, $k=2$ is used when classifying two material classes and $k=3$ is used when classifying three material classes.

The clustering algorithm is implemented as follows. First, each observation $\boldsymbol{o}$ is classified to the nearest centroid $\boldsymbol{\mu}_{j}$ using a distance metric such as Euclidean distance $\left\|\boldsymbol{o}_{i}-\boldsymbol{\mu}_{j}\right\|^{2}$. After all $n$ observations are classified, the centroids are recomputed as the means of their clusters $\mu_{j}=\frac{1}{N_{j}} \sum_{i=1}^{N_{j}} \boldsymbol{o}_{i}$, where $N_{j}$ is the number of observations belonging to the cluster $j$. The algorithm is iterated until no observation reassignments are made and the centroids stop moving.
TABLE I: Description of signals highlighted in Fig. 1.

\begin{tabular}{|r|l|l|}
\hline Signal & Description & Range \\
\hline \hline$\theta_{l}$ & lift cylinder extension & {$[0,604] \mathrm{mm}$} \\
\hline$\theta_{d}$ & dump cylinder extension & {$[0,396] \mathrm{mm}$} \\
\hline$u_{l}, u_{d}$ & lift $(l)$ and dump $(d)$ valve commands & {$[-1,1]$} \\
\hline$u_{t}$ & throttle command & {$[0,1]$} \\
\hline$v$ & wheel velocity & {$[0,3] \mathrm{m} / \mathrm{s}$} \\
\hline $\mathbf{a}_{1}, \mathbf{a}_{2}, \mathbf{a}_{3}$ & IMU1, IMU2 and IMU3 accelerations & {$[-24,24] \mathrm{g}$} \\
\hline
\end{tabular}

In this work, clustering performance is defined in terms of cluster purity $\bar{A} \in[0,1]$, where each cluster $\omega_{k}$ is assigned a class $c_{j}$ that is most frequent in that cluster, and the accuracy of this assignment is measured by counting the number of correctly assigned class members in each cluster and dividing by the total number of samples [11]. Thus, more formally

$$
\bar{A}(\boldsymbol{\Omega}, \boldsymbol{C})=\frac{1}{n} \sum_{k=1}^{K} \max _{c_{j} \in \mathbf{C}}\left|\omega_{k} \cap c_{j}\right|,
$$

where $\boldsymbol{\Omega}=\left\{\omega_{1}, \omega_{2}, \ldots, \omega_{K}\right\}$ is the superset of clusters and $\boldsymbol{C}=$ $\left\{c_{1}, c_{2}, \ldots, c_{J}\right\}$ is the superset of classes.

\section{FIELD EXPERIMENTS}

Data for material classification were acquired from manual excavation experiments by using a wheel loader and three different material piles. This section describes the experiment setup and excavation trials procedure.

\section{A. Robotic Loader Description}

The equipment used for field experiments was an instrumented Kubota R520s wheel loader. An image of the loader and its relevant sensors and actuators is shown in Fig. 1. This loader has a 1-tonne loading capacity and it is equipped with a custom bucket that is similar in design to buckets found on typical mining equipment (e.g., underground load-haul-dump machines). A summary of the signals used for this work are provided in Table I. The relevant systems and hardware are described below.

1) Cylinder Extension: Two wire potentiometers measure the lift cylinder extension $\theta_{l}$ and dump cylinder extension $\theta_{d}$.

2) Proportional Control Valves: Two electrohydraulic proportional valves control the fluid flow to the lift cylinders and the dump cylinder. The flow rates were proportional to the command signals $u_{l}=[-1,1]$ and $u_{d}=[-1,1]$ for the lift cylinders and the dump cylinder, respectively. Here, negative command values corresponded to cylinder retraction and positive command values corresponded to cylinder extension. The command signals were sent by the joystick during manual operation.

3) Throttle: The throttle pedal is actuated by a linear servo motor. The position command to this motor $u_{t}$ is a value between 0 and 1 , where $u_{t}=1$ corresponds to a fully depressed throttle. Engine RPM is not logged in the current setup, but the tachometer value can be read by the operator by viewing a display on the dashboard. 


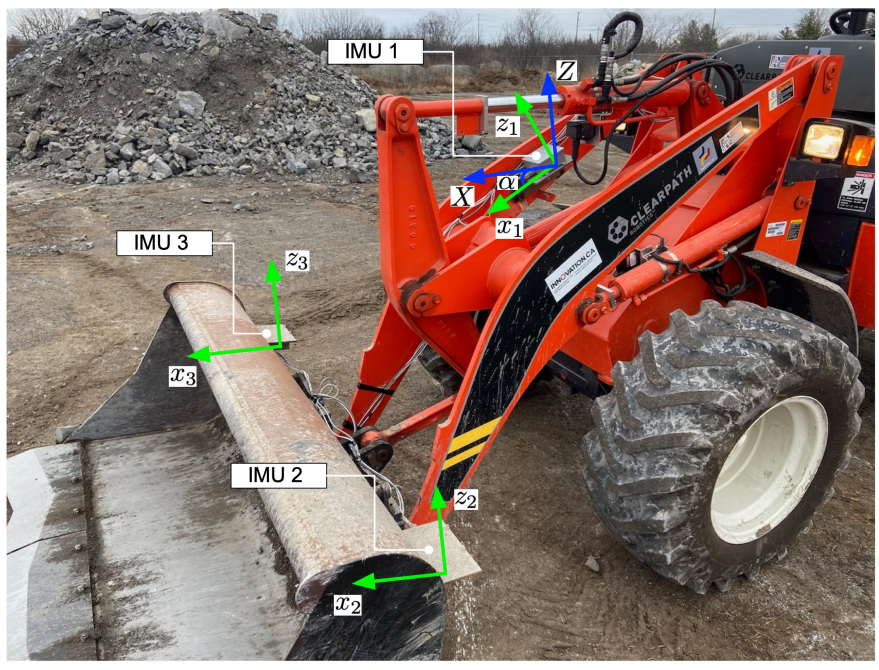

Fig. 2: Installed locations and orientations of the three IMUs. Note that the body coordinate frame $(X, Z)$ is fixed to the front of the vehicle (shown better in Fig. 1), so $X$ is parallel to the ground.

4) Wheel Encoders: Two encoders attached to the front wheels provide the wheel speed measurement $v$.

5) Inertial Measurement Units (IMU): Three 6-axis Bosch BMI088 IMUs were installed on the boom and bucket to acquire acceleration measurements $\mathbf{a}=\left[a_{x}, a_{y}, a_{z}\right]$ during loading; the gyroscopes were not utilized in this work. During the loading experiments with the small scale haul truck [8], it was observed that the IMU mounted directly to the truck bed measured acceleration signals with larger amplitude compared to the IMU mounted to the chassis. These results informed the placement of IMUs on the bucket and the boom in this preliminary work. The locations and orientations are shown in Fig. 2. IMU 1 was installed on the boom, IMUs 2 and 3 were installed on the left and right side of the bucket respectively. IMU 1 is oriented such that its $x$-component is along the boom arm. IMU 2 and IMU 3 were oriented such that their $x$-components are parallel to the base of the bucket. Each IMU was enclosed within an IP67-rated enclosure. The two enclosures on the bucket were mounted beneath thick metal brackets to protect the IMUs from falling rocks.

6) Control System: An onboard control system was used to $\log$ sensor data and the actuator command signals. An overview of the hardware architecture is shown in Fig. 3. The control system consists of a main control unit (MCU), seven CAN Peripheral Interface (CPI) sub-control modules, and a laptop running the Robot Operating System (ROS). The MCU operated at $10 \mathrm{~Hz}$ for logging sensor data and sending commands via the CPIs. The three IMUs and the joystick were connected through Arduino® microcontrollers to ROS, which logged the acceleration data at $100 \mathrm{~Hz}$.

\section{B. Rock Piles}

Three rock piles with different rock size distributions were used for the loading experiments. Sample images from the

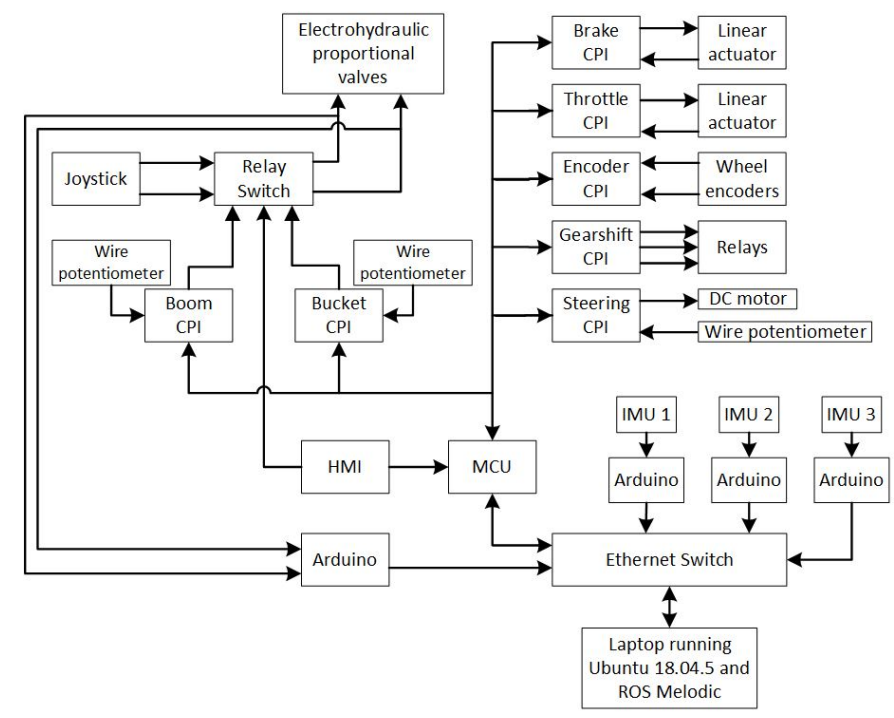

Fig. 3: Overview of the loader hardware architecture.

three piles are shown in Fig. 4. Each pile contained approximately $35 \mathrm{~m}^{3}$ of material. The material properties are described in further detail below.

1) Rock: The Rock pile, shown in Fig. 4a, contained a large distribution of fragmented limestone, ranging from fine particles less than $1 \mathrm{~mm}$ to large rocks that were $600 \mathrm{~mm}$ along the longest length.

2) Granular B: The Granular B pile, shown in Fig. 4b, was obtained from an aggregate quarry. The quarry routinely performs size distribution testing on their products to ensure conformance to government standards for aggregates [12]. The size distribution produced by the quarry estimated a maximum particle size of $75 \mathrm{~mm}$, however particles up to $120 \mathrm{~mm}$ in size can be observed from Fig. 4b. This discrepancy in maximum size could be attributed to the relatively small sample $(30 \mathrm{~kg})$ used for size distribution estimation.

3) Granular A: The Granular A pile, shown in Fig. 4c contains the smallest particle sizes out of the three materials. No size distribution was available for this pile. Through inspection and hand measurements taken during the experiments, the maximum rock size for Granular A was found to be approximately $25 \mathrm{~mm}$.

\section{Excavation Trial Procedure}

Each manual excavation trial was performed by the following steps:

1) Move the lift and dump cylinders to pile entry position $\left(\theta_{l}=16 \mathrm{~mm}, \theta_{d}=272 \mathrm{~mm}\right)$. The pile entry configuration is shown in Fig. 1.

2) Set the throttle input $u_{t}=0.8$ (verify that engine tachometer reads 2200 RPM).

3) Start the data logging.

4) Engage the forward gear and release the brakes.

5) After pile is penetrated, manually dig and load material. Note that only the the dump cylinder is actuated during this step. 


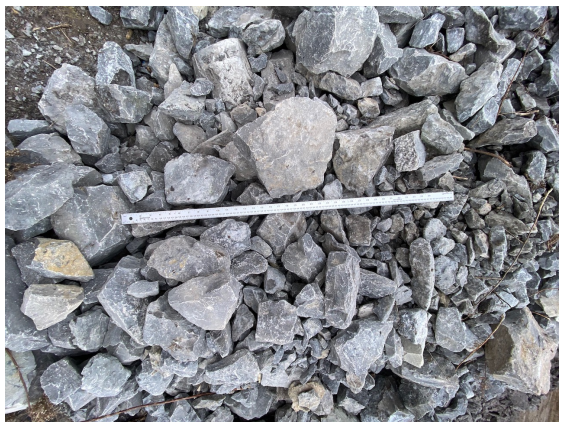

(a) Rock

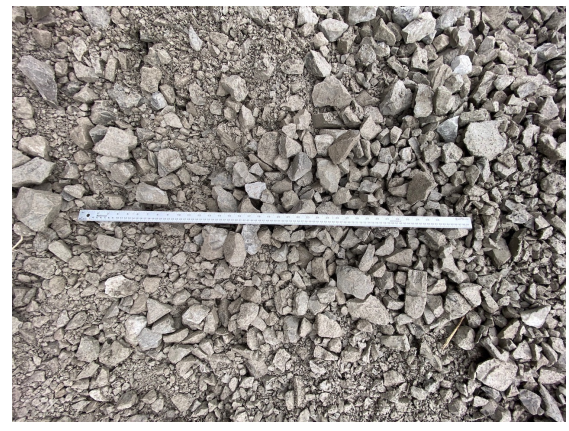

(b) Granular B

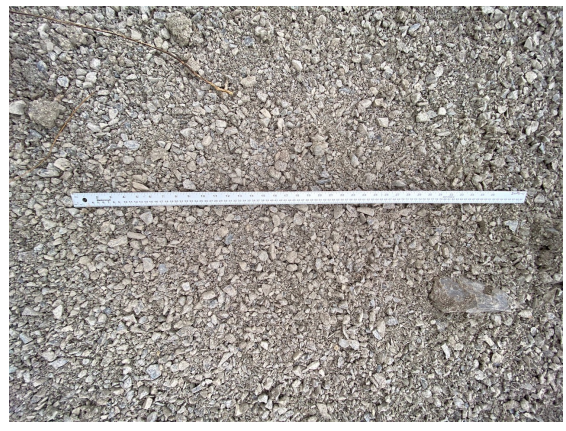

(c) Granular A

Fig. 4: The three materials that were used for manual loading experiments. A $1 \mathrm{~m}$ ruler is shown for scale.

6) When the bucket is fully curled, stop digging and loading.

7) Reverse the loader out of the pile.

8) Extend the lift cylinders to $\theta_{l}=516 \mathrm{~mm}$. This is done to weigh the material.

9) Stop the data logging.

10) Dump material back onto the pile.

\section{Data Set and Signal Processing}

Field experiments with the loader are very time consuming and require careful preparation. A total of 11 trials with the rock pile, 14 trials with the Granular B pile and 7 trials with the Granular A pile constitute the data set for the classification results presented in this paper. The following subsections present the signal processing techniques implemented to prepare the feature set for classification.

\section{A. Acceleration Signals}

For this study, only one component from each IMU was used for wavelet feature extraction and analysis. These three accelerations included $a_{2, x}$ and $a_{3, x}$ from IMU 2 and IMU 3, respectively, and the transformed acceleration component

$$
a_{1, X}=a_{1, x} \cos \alpha-a_{1, z} \sin \alpha
$$

from IMU 1 in the body frame of the loader $(X, Z)$ as shown in Fig. 2. The rotation angle $\alpha$ was estimated using

$$
\alpha=\tan ^{-1}\left(\frac{\bar{a}_{1, x}}{\bar{a}_{1, z}}\right),
$$

where $\bar{a}_{1, x}$ and $\bar{a}_{1, z}$ are the mean acceleration values over the first $1 \mathrm{~s}$ of data (i.e., between Step 3 and Step 4 in Section III-C). This transformation reduces the impact of different boom IMU orientations due to errors in positioning the lift cylinder in the pile entry position (Step 1). With this transformation, the acceleration in the $X$-direction in the body coordinate frame as shown in Fig. 1 was used for IMU 1.

An example of the captured accelerometer data along with the dump cylinder command inputs is presented in Fig. 5.
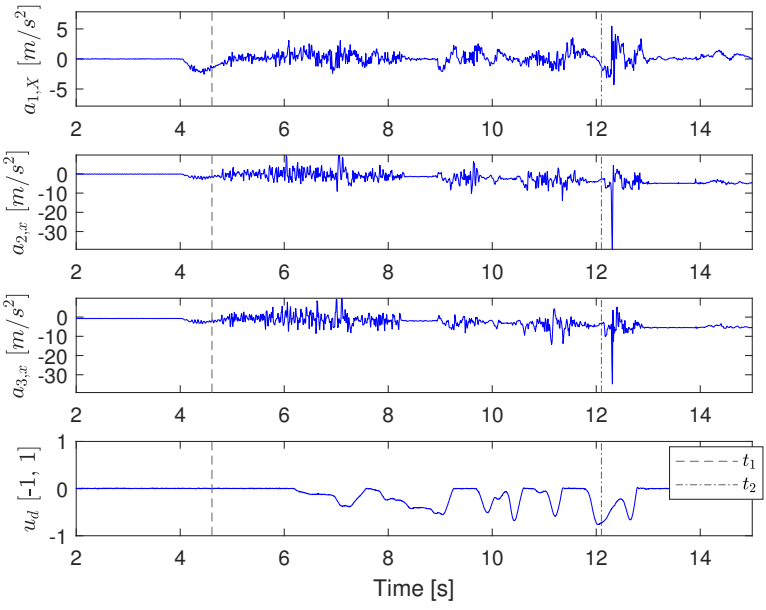

Fig. 5: An example acceleration signals and dump cylinder command inputs.

\section{B. Excavation Start Time}

The start time, $t_{1}$, of the excavation cycle was identified as the time where the derivative of the accelerometer signals, $|\dot{a}|$, exceeds a threshold, $\gamma$. When the loader first moves forward, Step 4 in Section III-C, the metric could potentially reach the threshold prematurely. To minimize the potential impact the search window starts $0.5 \mathrm{~s}$ after the loader started to move forward by inspection of the velocity estimate $v$. An example start time using the developed process is given in Fig. 6. Through inspection of multiple trials, $\gamma$ was found to be $45 \mathrm{~m} / \mathrm{s}^{3}$.

\section{Excavation Stop Time}

The end of the excavation cycle, $t_{2}$, was estimated using the dump cylinder extension, $\theta_{d}$. The excavation cycle ends when the dump cylinder reaches the minimum of either 372 $\mathrm{mm}$ or when the dump cylinder reaches the extension value $15 \mathrm{~s}$ after $t_{1}$. The first criteria ensures the induced vibration caused by the bucket reaching the mechanical stops is not contained within the excavation window. The second criteria 

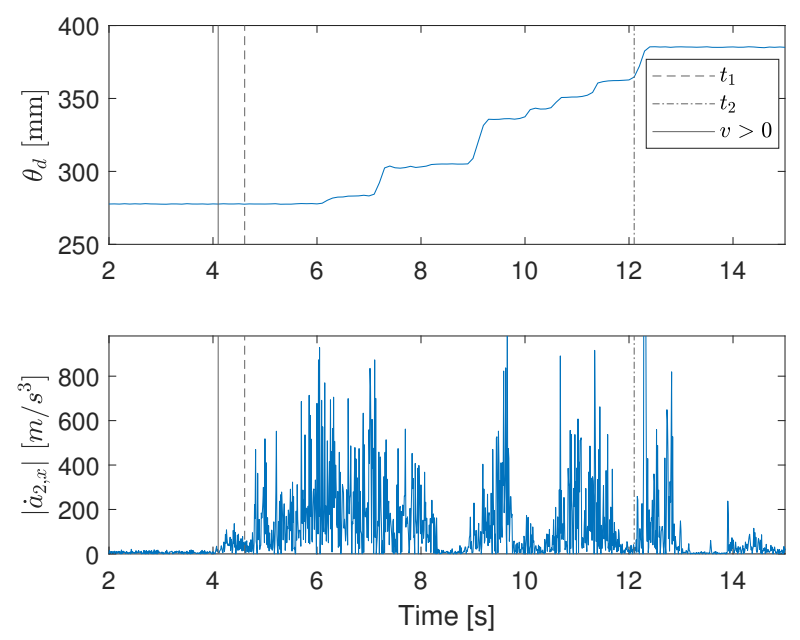

Fig. 6: An example of calculating $t_{1}$ and $t_{2}$ using $|\dot{a}|$ and $\theta_{d}$, respectively.

overcomes the scenario when the operator fails to extend the dump cylinder fully during excavation.

\section{RESULTS}

The material classification methodology was tested by first extracting wavelet features described in Section II-A from the segmented acceleration signals $a_{1, X}, a_{2, x}$ and $a_{3, x}$. The features were then clustered using the $k$-means algorithm described in Section II-B to test the classification performance for the three material classes. MATLAB ${ }^{\circledR} 2020$ b (version 9.9) with Wavelet Toolbox (version 5.5) and Statistics and Machine Learning Toolbox (version 12.0) were utilized for this analysis. The following subsections present the feature extraction and classification results.

\section{A. Feature Extraction using Wavelet Analysis}

To perform the feature extraction, the $\mathrm{Cwt}^{1}$ function, which is available in the Wavelet Toolbox, was first used to apply the continuous 1-D wavelet transform (CWT), using (1) and (2), to the acceleration signals. Next, the resulting $C W T(f, \tau)$ was integrated over time, using (3), to obtain for $\beta(f)$ each signal. Finally, $\beta(f)$ was normalized by the dig time $T=t_{2}-t_{1}$ for each signal. The normalized result, $\beta(f)$, was resampled starting at $1.5 \mathrm{~Hz}$, with $1 \mathrm{~Hz}$ increments, and stopping at $36.5 \mathrm{~Hz}$. Thus, $n=36$ features were extracted from each acceleration signal. These features were stored as $\boldsymbol{o}_{36 \times 1}$ feature vectors to use as inputs for the classification algorithm. Note that the $1 \mathrm{~Hz}$ increment value is tunable and $1 \mathrm{~Hz}$ captured the low frequency fluctuations while not oversampling the higher frequencies.

The features $\beta(f)$ extracted from $a_{1, X}, a_{2, x}$ and $a_{3, x}$ for the 32 excavation trials are shown in Fig. 7, Fig. 8 and Fig. 9 , respectively. At higher frequencies the features appear to distinguish the Granular A pile from the Rock and Granular

\footnotetext{
${ }^{1}$ https://www.mathworks.com/help/wavelet/ref/cwt.html
}

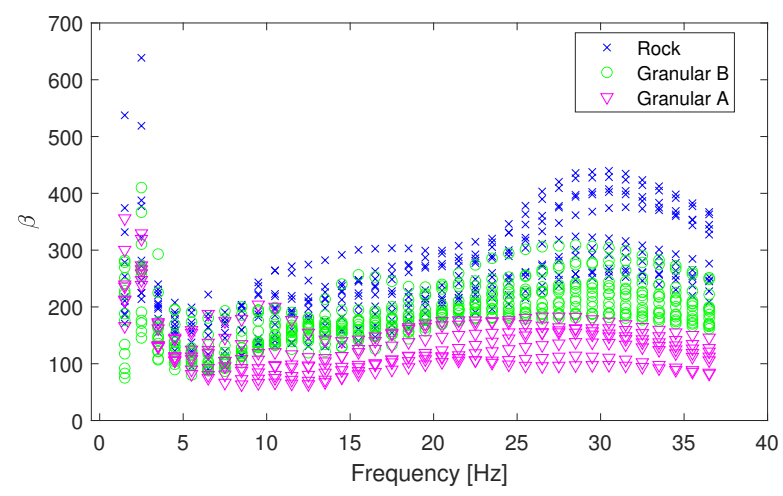

Fig. 7: Wavelet feature set extracted from $a_{1, X}$.

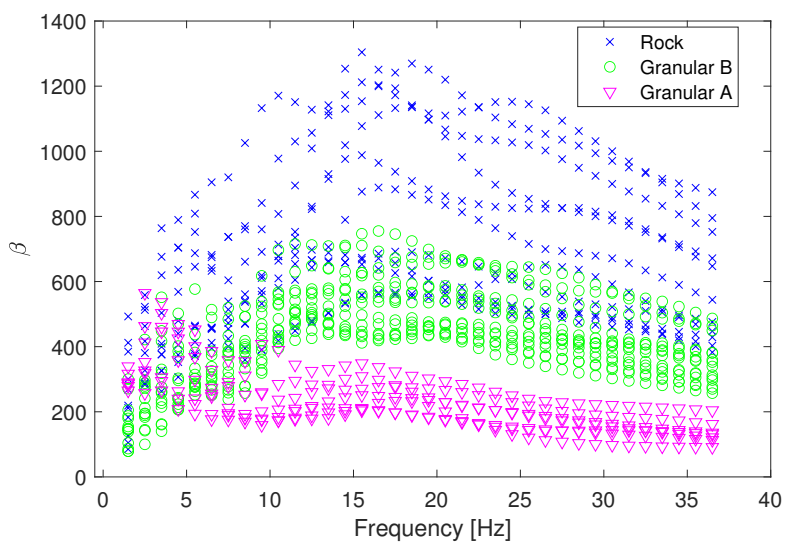

Fig. 8: Wavelet feature set extracted from $a_{2, x}$.

B; however Rock and Granular B have some overlap. There does not exist one frequency that can distinguish all three piles. The overlap at lower frequencies could arise due to each pile having a portion of smaller sized particles.

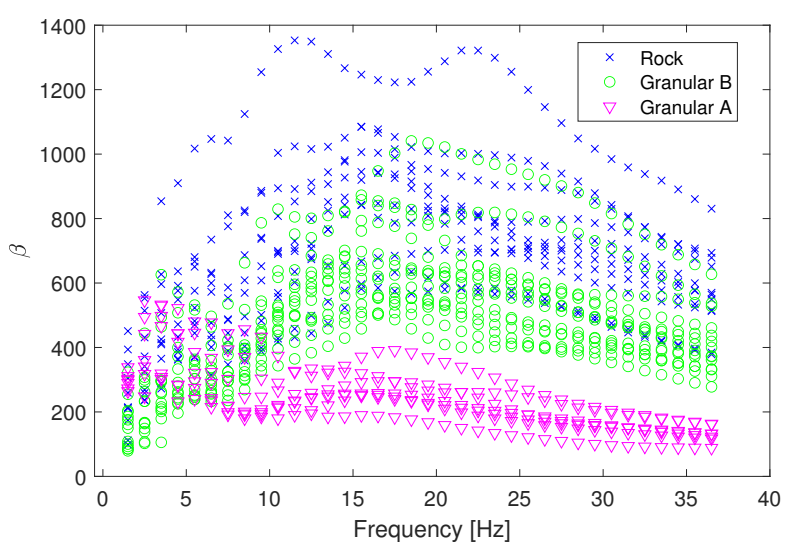

Fig. 9: Wavelet feature set extracted from $a_{3, x}$. 
TABLE II: Classification accuracy.

\begin{tabular}{|c|c|c|c|}
\hline Pile ID & $a_{1, X}(\%)$ & $a_{2, x}(\%)$ & $a_{3, x}(\%)$ \\
\hline \hline Rock \& Gran. B & 92 & 80 & 80 \\
\hline Rock \& Gran. A & 93 & 86 & 92 \\
\hline Gran. B \& Gran. A & 71 & 86 & 77 \\
\hline Rock \& Gran. B \& Gran. A & 86 & 83 & 75 \\
\hline
\end{tabular}

\section{B. Classification using k-means}

The $n=32$ feature vectors $\boldsymbol{o}$ were clustered using the $k$ means unsupervised learning algorithm. The algorithm was initialized with $k=3$ prototypes to form 3 clusters with the feature set when comparing Rock, Granular B and Granular A and initialized with $k=2$ prototypes for binary classification.

The feature sets from the three different acceleration signals are classified independently. In a practical implementation, the fewest number of sensors are preferred to reduce costs and complexity which is the motivation for each signal processed independently.

For each feature set, binary classifications of two different materials, as well as the full classification of all three materials was performed. Binary classification assessed the performance of the features from piles with similar size distribution.

The $k$-means classification algorithm with $k=3$ and $\beta(f)$ as inputs is used to classify the piles. The classification performance using (4) of different combinations of the three piles is given in Table II.

Table II reveals that the classification rates are similar with the boom mounted IMU performing slightly better than the two bucket IMUs. The different classification accuracy from the two bucket IMUs is somewhat concerning, and may imply a hardware issue. Rock and Granular A classification obtained the highest result likely because these two piles represent the largest difference in maximum rock size.

\section{Discussions}

Although our methodology achieved high classification accuracies, some discussion items arise. The features were created by using only the forward direction ( $X$ for IMU $1, x$ for IMUs 2 and 3) accelerations where the orthogonal component $Z$ for IMU 1 and $z$ for IMUs 2 and 3 could also be used in the classifier. The lower classification accuracies of the bucket IMUs could indicate that using the data in the local coordinate system may lead to poorer outcomes. The lower classification accuracy of IMU 3 may indicate a potential hardware issue which requires further investigation. The observed variability in the wavelet features for each pile could also be attributed the human operator's skill level, where a more experienced operator may yield more consistent excavations which should produce a higher classification accuracy. Normalization using payload (amount of material in the bucket after excavation) could potentially be useful.

All 36 features were used as inputs to the classification algorithm and feature size reduction was not investigated. The motivation was to highlight possible trends in the results that could potential correlate to the different size distributions. As the end goal is to develop a system that outputs size distribution model parameters the results are a good first step towards this end.

In some applications, the likelihood of equipment having sensors that measure both the cylinder extensions and wheel velocity is low. One possible method to remove the need for these two inputs could be to start the loader at a certain distance from the pile and to pause for a small duration after excavation is completed.

\section{CONCLUSIONS}

This paper demonstrates the extension of preliminary research [8] using accelerometer data combined with wavelet analysis to distinguish between rock piles of different size distributions during the excavation process. An instrumented 1-tonne capacity loader was manually operated to perform excavation using three rock piles with different rock size distribution. The developed method was able to classify the different rock piles with an average of $81 \%$, despite the variability introduced by manual excavation. As a next step, we hope to extend this method to estimate size distribution model parameters compared to the discrete pile classification demonstrated here.

\section{REFERENCES}

[1] A. Beyglou, D. Johansson, and H. Schunnesson, "Target fragmentation for efficient loading and crushing - the aitik case," Journal of the Southern African Institute of Mining and Metallurgy, vol. 117, pp. 10531062, 2017.

[2] F. Ouchterlony and J. Sanchidrian, "A review of development of better prediction equations for blast fragmentation," Journal of Rock Mechanics and Geotechnical Engineering, vol. 11, no. 5, pp. 1094-1109, 2019.

[3] H. A. Fernando, J. A. Marshall, and J. Larsson, "Iterative learning-based admittance control for autonomous excavation," Journal of Intelligent \& Robotic Systems, vol. 4, no. 3, pp. 495-500, 2019.

[4] H. A. Fernando and J. A. Marshall, "What lies beneath: Material classification for autonomous excavators using proprioceptive force sensing and machine learning," Automation in Construction, vol. 119, p. 103374,2020 .

[5] S. Tafazoli Bilandi, M. Ramezani, I. L. Bell, M. Sameti, P. Abolmaesumi, and S. Nouranian, "Method and apparatus for identifying fragmented material portions within an image," World Intellectual Property Organization (WIPO) Patent No. WO2017100903A1, June 22, 2017.

[6] T. Bamford, K. Esmaieli, and A. Schoellig, "A real-time analysis of postblast rock fragmentation using UAV technology," International Journal of Mining Reclamation and Environment, vol. 31, pp. 439-456, 062017.

[7] C. McKinnon and J. A. Marshall, "Automatic identification of large fragments in a pile of broken rock using a time-of-flight camera," IEEE Transactions on Automation Science and Engineering, vol. 11, no. 3, pp. 935-942, 2014.

[8] U. Artan and J. Marshall, "Towards automatic classification of fragmented rock piles via proprioceptive sensing and wavelet analysis," in Proceedings of the 2020 IEEE Conference on Multisensor Fusion and Integration for Intelligent Systems (MFI), 2020, pp. 348-353.

[9] A. K. Louis, Wavelets: theory and applications, ser. Pure and applied mathematics. Chichester: Wiley, 1996.

[10] R. O. Duda, P. E. Hart, and D. G. Stork, Pattern Classification, 2nd ed. New York: John Wiley \& Sons, Inc, 2001.

[11] C. D. Manning, P. Raghavan, and S. H., Introduction to Information Retrieval, 1st ed. New York: Cambridge University Press, 2008.

[12] “OPS Complete Manual Vol 6," https://www.library.mto.gov.on.ca, pp. 53-68, Apr 2020. 